\title{
Codierung und Analyse der Sprachinhalte Klinischer Interviews
}

Barbara Winkler und Heiner Ellgring, München

Einführung und Problemstellung:

Gesprochene Sprache bildet einen wesentlichen Teil menschlichen Handelns. Aus dem, was ein Sprecher oder Kommunikator sagt (und daraus, wie er es sagt) schließt ein Hörer oder Interpret $u$. a. darauf, was der andere ihm mitteilen will, was ihn beschäftigt, wie er sich selbst und seinen Gesprächspartner sieht, und was er, der Hörer (Interpret) nun tun solle. In ihrem Handeln werden beide - Kommunikator und Interpret - maßgeblich durch die gesamte Kommunikationssituation und Kommunikationsintention geleitet (Fearing, 1967).

Dieser Gesichtspunkt ist besonders im Hinblick auf die uns interessierenden Gespräche zwischen einem Kliniker (Psychiater oder Psychologen) und einem Patienten zu berücksichtigen, denn nicht zuletzt schließt hier der Hörer oder Interpret über den Kommunikationsinhalt auf den psychopathologischen Zustand des Sprechers oder Kommunikators. Zum anderen wird das, was der Sprecher produziert, entscheidend mitbestimmt durch das Verhalten seines Partners (Matarazzo, Wiens \& Saslow, 1965).

Die folgende Untersuchung konzentriert sich auf den inhaltlichen Aspekt der Kommunikation zwischen Kliniker und Patient. Dabei sollen die inhaltlichen Merkmale der verbalen Interaktion zwischen den beiden Teilnehmern nicht nur qualitativ beschrieben, sondern auch quantitativ erfaßt werden. Wir benötigen dazu ein Instrument, das in der Lage ist, über das Sprechverhalten und seine Veränderungen in der Zeit Zustandsänderungen der Gesprächsteilnehmer zu erfassen. Hier interessieren besonders Veränderungen im Zustand des Patienten.

Weiterhin soll die Art der Interaktion zwischen Kliniker und Patient in groben Zügen beschrieben werden.

Bisherige Ansätze zur Analyse von Sprechverhalten im klinischen Bereich verwendeten häufig mehrminütige Sprachproben der Patienten ohne Kommunikationspartner. Die Patienten wurden dabei aufgefordert, über selbstgewählte Themen frei ins Mikrophon zu sprechen (z. B. Weintraub \& Aronson 1967, Gottschalk \& Gleser 1969). Und auch dann, wenn die Sprachproben aus Interviews zwischen Kliniker und Patient stammten, wurde in der Regel nur das Sprechverhalten des Patienten und nicht das des Partners untersucht (z. B. Mahl 1965, Lindenfeld 1972).

$\mathrm{Da}$ wir speziell an der Interaktion depressiver Patienten und ihrer Gesprächspartner interessiert sind, sollte ein Codierungssystem entwickelt werden, das folgenden Anforderungen genügt: 
a) Vermutlich depressionsspezifische Merkmale im Sprachverhalten sollen erfaßt werden, wie z. B. die Frage, ob bestimmte thematische Inhalte gehäuft auftreten, und wie diese sich ändern (vgl. Howard et al. 1969, Weissmann \& Bothwell 1967). Zudem sollte gleichzeitig u. a. der Frage nachgegangen werden, ob die negative Bewertung, die laut Beck (1967) depressive Patienten in ihrem Selbstkonzept, ihrer gesamten Weltsicht und $\mathrm{Zu}$ kunftseinschätzung vornehmen, über unser Analysematerial nachweisbar ist, und wie sich dieser Aspekt möglicherweise verändert.

b) Zum anderen sollten Phänomene untersucht werden wie die Aufeinanderfolge und gegenseitige Bezugnahme der Außerungen der Gesprächspartner, und auch der formale Gesprächsstil, um damit spezielle Merkmale der Interaktion zwischen Kliniker und Patient zu analysieren.

Beschreibung des Codierungssystems:

In unserem Codierungssystem werden nun folgende acht Merkmale berücksichtigt:

1. Thematik

2. Ich-Beteiligung

3. Aktivitätsgrad

4. Zeitperspektive

5. Implizierte Bewertung

6. Ausgedrückte Emotion

7. Bezug zur đußerung des Gesprächspartners

8. Äußerungsform

Für jedes Merkmal existiert eine Anzahl von Kategorien, die je nach Merkmal variiert. Hier sollen vier der Merkmale etwas detaillierter beschrieben werden. Das Merkmal 1 ,Thematik“ hat z. B. folgende Kategorien:

1.1 Sozialer Kontakt; 1.2 Beruf, Leistung, Konzentration, Lebensbewältigung; 1.3 Ökonomische Lage; 1.4 Gedanken, Vorstellungen, Träume, Gefühle; 1.5 Körperliches Befinden, Gesundheit, Krankheit; 1.6 Behandlung, Behandlungsvorschriften, - effekte; 1.7 Klinikaufenthalt.

Die Operationalisierung von 1.2 sieht dabei folgendermaßen aus: „es wird gesprochen über Berufsarbeit, Vorkommnisse an der Arbeitsstelle, Leistung, Leistungsversagen, Konzentration und damit zusammenhängende Fragen, sowie Probleme der allgemeinen Lebensführung und Lebensbewältigung“.

Das Merkmal 5 „Implizierte Bewertung“" ist untergliedert in 5.1 positiv, 5.2 negativ, 5.3 neutral. „Negativ“ (5.2) wird codiert, wenn in die Äußerung eine negative Bewertung (z. B. der eigenen Person, der Umwelt, der Zukunftsmöglichkeiten) eingeht. Der Sprecher macht sich Vorwürfe, sieht sich als „,schuldig“, „hoffnungslosen Fall“".

Mit Hilfe der Subkategorien von Merkmal 7 „Bezug zur Äußerung des Gesprächspartners" wird codiert, in welcher Art die Äußerungen der Gesprächspartner aufeinander bezogen sind. 7.1 wird codiert, ,,wenn aus der Äußerung ein formal deutlicher Bezug zu jener des Gesprächspartners (GP) erkennbar ist. Bei diesem Bezug kann es sich um eine Konkretisierung des vom GP An- 
gesprochenen handeln, oder auch um einen vagen, unbestimmten Bezug zu jenem."

Weiterhin werden alle Äußerungen der Gesprächspartner mittels der Unterpunkte von Merkmal 8 „Außerungsform" global danach codiert, ob es sich bei der Äußerung um eine Aussage oder um eine Frage handelt. Fine Codierung mit 8.2 „Frage“ wird z. B. dann vorgenommen, wenn ,,in der Außerung eine Bitte um Information etc. enthalten ist. 2 wird auch codiert, wenn die Frage erst am Ende einer längeren Außerung auftaucht."“

\section{Datengewinnung:}

Im folgenden werden Daten berichtet aus einer Verlaufsuntersuchung an einem Patienten mit der Diagnose ,endogene Depression“. Mit Hilfe des Codierungssystems werden jeweils die ersten fünf Minuten eines freien Gespräches mit einem Kliniker untersucht. Die Gespräche schließen sich an routinemäßig zweimal wöchentlich durchgeführte Standardinterviews an. Derartige Interviews werden während des gesamten stationären Aufenthaltes des $\mathrm{Pa}$ tienten geführt, ebenso zum Zeitpunkt der Nachuntersuchung, zu der der $\mathrm{Pa}$ tient einige Monate nach seiner Entlassung nochmals für etwa 14 Tage in die Klinik kommt. In einem Studio werden diese Gespräche video-aufgezeichnet, wobei beide Gesprächsteilnehmer darüber informiert sind, daß sie aufgenommen werden. Aus der Gesamtmenge der Interviews mit dem Patienten wurden fünf ausgewählt, und zwar aus dem Beginn des Klinikaufenthaltes, einer Zeit, in der der Patient sein subjektives Befinden als schlecht angibt, aus der Zeit der Besserung des subjektiven Befindens, der Zeit kurz vor der Entlassung und zum Zeitpunkt der Nachuntersuchung.

Von den ersten fünf Minuten der freien Gesprächssituation nach den Standardfragen werden jeweils wortgetreue Transkripte angefertigt. Sie beginnen mit der Eröffnungsfrage des Klinikers: ,Wie glauben Sie, wird es auf längere Sicht mit Ihnen weitergehen?"،

Die Anwendung unseres Codierungssystems auf das transkribierte Material geschieht nun in folgender Weise: Die erste Äußerung des Interviewers (nämlich die zitierte offene Frage) erhält die Kennziffer K1. Die nachfolgenden Außerungen des Patienten die Kennziffer P1, darauf K2 usw.. Auch kurze Einwürfe in den Gesprächsfluß des anderen, z. B. Rückmeldungen wie ,hm“ und ,ja“" werden als Äußerungen gekennzeichnet. Die so bezeichnete Äußerung jedes einzelnen Gesprächspartners bildet gleichzeitig eine Analyseeinheit. Mit dieser Art der Segmentierung des transkribierten Sprachmaterials schließen wir uns dem Vorgehen anderer Autoren an, wie Mintz \& Luborsky (1970) und Brenner \& Hjelmquist (1978). Für jede Äußerung der beiden Gesprächsteilnehmer wird nun in jedem Merkmal des Codierungssystems die geeignete Kategorie bestimmt.

Als quantitative Größe werden die Häufigkeiten der Merkmalskategorien bestimmt, relativiert auf die Anzahl der Außerungen eines Interaktionspartners. 
Ergebnisse:

Die Gesamtäußerungsmenge bewegte sich zwischen 29 Äußerungen pro Gesprächsteilnehmer im ersten der untersuchten Interviewausschnitte und 57 Außerungen in dem Gesprächsausschnitt zum Zeitpunkt der Nachuntersuchung.

Die weiter unten aufgeführten Häufigkeitsänderungen sollten immer in Beziehung zu den in Abb. 1 dargestellten subjektiven Veränderungen im Zustand des Patienten gesehen werden.

In Abbildung 1 ist das subjektive Befinden des Patienten als Wert in einem „Zustandsbarometer" angegeben.

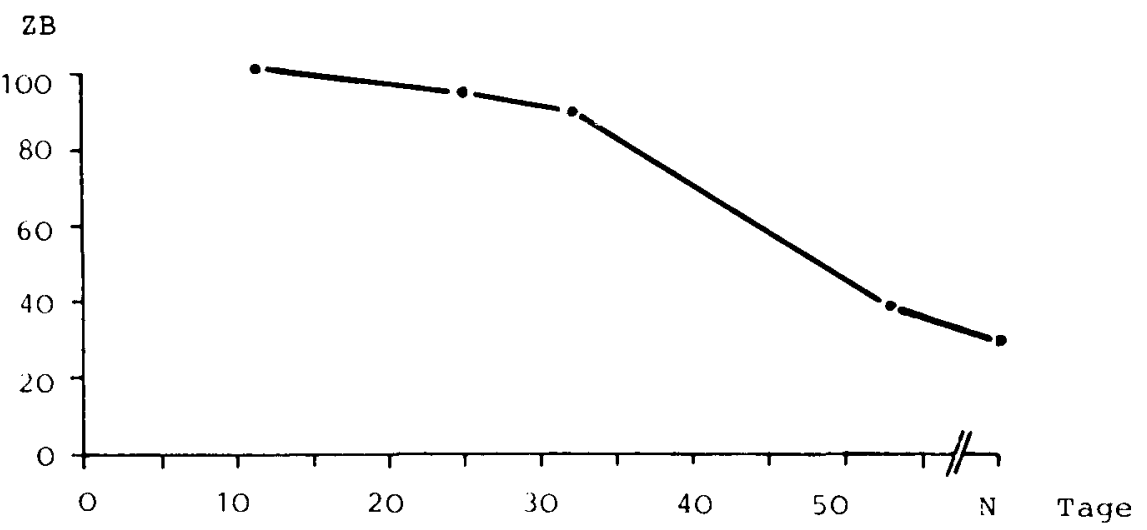

Abb. 1: Verlauf des selbsteingeschätzten subjektiven Befindens des Patienten.

Ein hoher Wert im ,Zustandsbarometer" (ZB) - Maximalwert 120 - bedeutet dabei, daß der Patient sein subjektives Befinden als schlecht einschätzt, ein niedriger Wert - Minimalwert 0 - heißt, daß er seinen Zustand als angenehm erlebt. Bei dem hier untersuchten Patienten ist erkennbar, daß sein so eingeschätztes Befinden sich über den Zeitverlauf langsam bessert. Die deutlichste Veränderung sehen wir zwischen dem dritten und vierten der von uns untersuchten Interviews.

Die Merkmalsveränderungen während des Klinikaufenthaltes und danach zeigen die folgenden Abbildungen. Der gleichzeitige Verlauf der Werte des Zustandsbarometers ist der gepunkteten Kurve zu entnehmen.

„Thematik":

Deutliche Verschiebungen über den Zeitverlauf werden im Merkmal „,Thematik" erkennbar. 


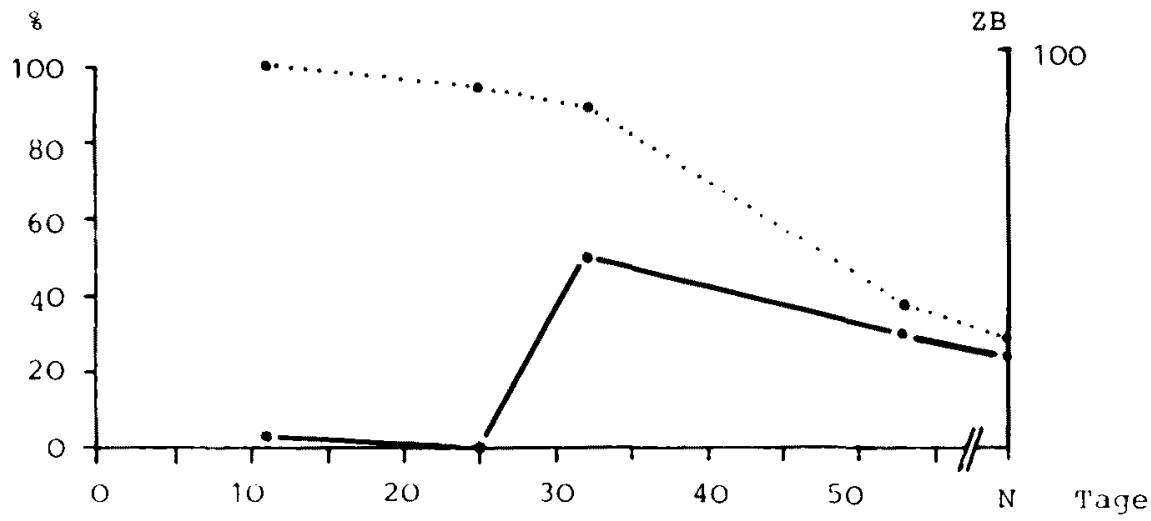

Abb. 2: Merkmal 1 „Thematik“ - relative Häufigkeit der Codierung von 1.1. ,Sozialer Kontakt'

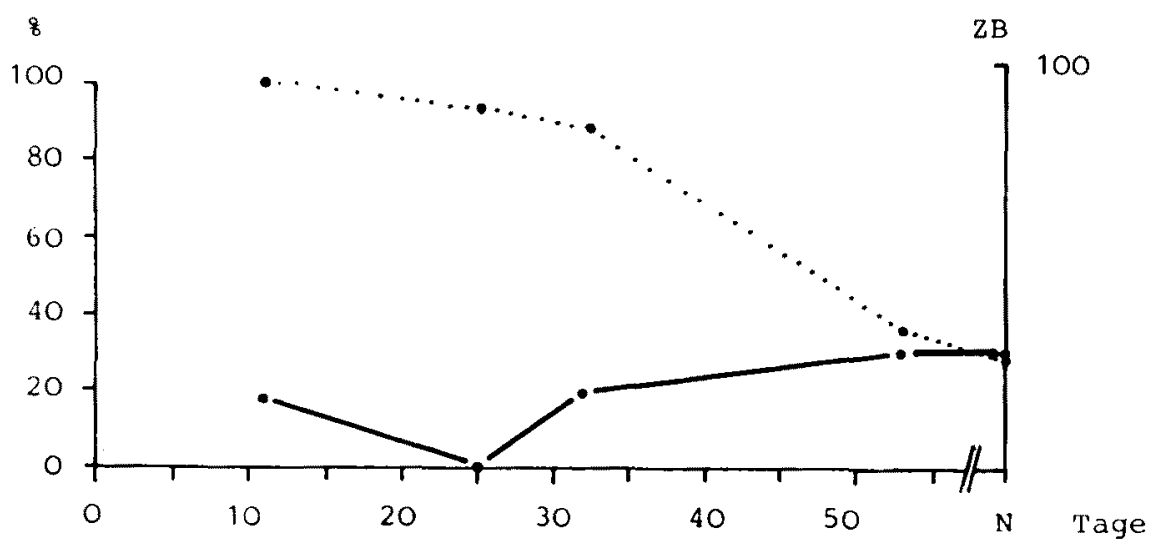

Abb. 3: Merkmal 1 "Thematik" - relative Häufigkeit der Codierung von 1.2. ,Beruf etc:"

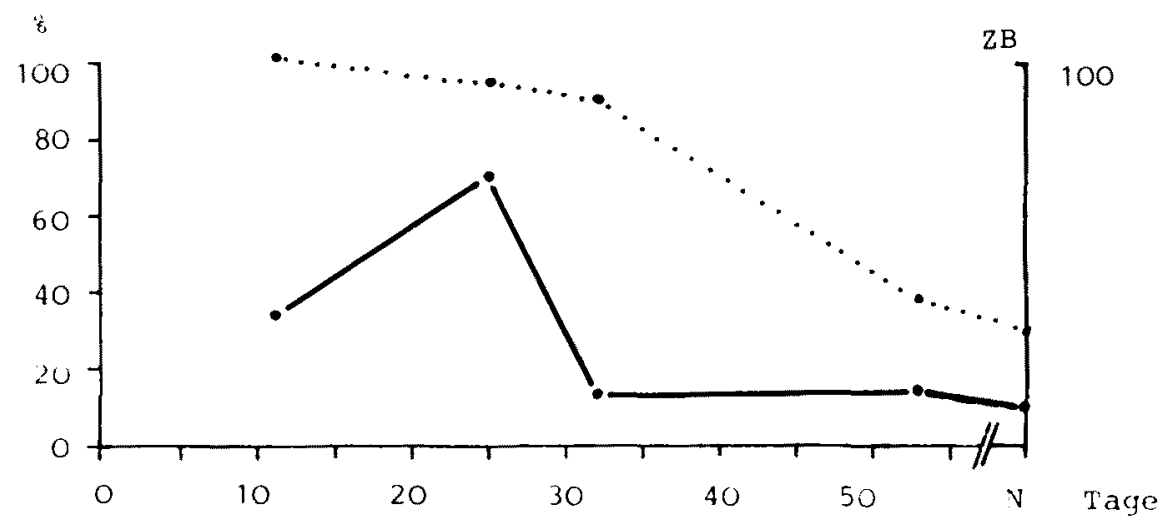

Abb. 4: Merkmal 1 „Thematik" - relative Häufigkeit der Codierung von 1.4. ,Gedanken etc." 
Das Thema „Sozialer Kontakt" wird in den beiden ersten Interviews vom Patienten nicht oder kaum angesprochen (Abb. 2). Im dritten Interview jedoch spielt „Sozialer Kontakt“" in der Hälfte der Außerungen eine Rolle und pendelt sich in den beiden letzten Interviews auf etwa ein Viertel der Äußerungen ein.

Die Thematik „Beruf, Leistung, Konzentration, Lebensbewältigung“ (Abb. 3) tritt in den Äußerungen des Patienten während des ersten und dritten Interviews etwa gleich häufig auf, während dieser Bereich im zweiten Interview gar nicht erwähnt wird. Im vierten und fünften Interview hingegen wird diese Thematik gleichbleibend in etwa $30 \%$ der Außerungen angeschnitten. (Im Zusammenhang mit dem Merkmal „Bewertung“ kommen wir auf dieses Ergebnis noch einmal zurück). Der Anteil von „Gedanken, Vorstellungen, Träumen, Gefühlen“ am Merkmalskomplex ,Thematik"schwankt in den verschiedenen Interviews und nimmt nach dem zweiten Gespräch deutlich ab.

„Bewertung":

Uber die verschiedenen Interviews nimmt der Patient deutliche Veränderung vor in der subjektiven Bewertung dessen, was er sagt.

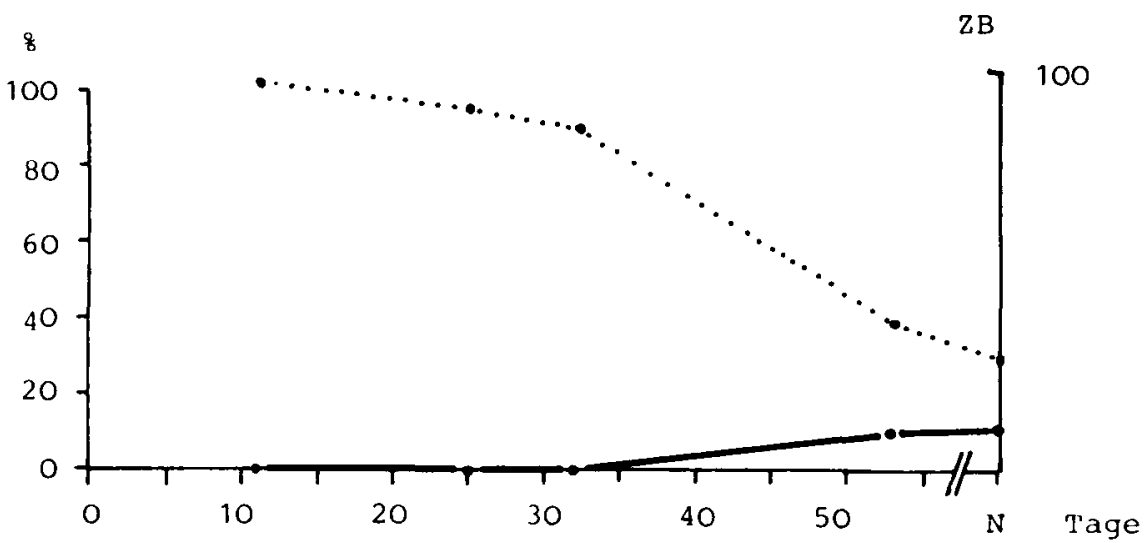

Abb. 5: Merkmal 5 „Implizierte Bewertung“ - relative Häufigkeit von ,positiver Bewertung"

Wie der Abbildung $5 \mathrm{zu}$ entnehmen ist, enthält keine der Äußerungen aus den ersten drei Interviews eine positive Bewertung des Gesagten. Erst in den beiden letzten Interviews finden sich in ca. $10 \%$ der Äußerungen positive Bewertungen.

Dagegen treten „Negative Bewertungen“ im ersten der untersuchten Interviews in gut $75 \%$ der Außerungen auf, im zweiten Interview nur noch $50 \%$ aller Äußerungen. In den letzten drei Interviews zeigen sich derartige negative Bewertungen kaum noch, oder gar nicht mehr (s. Abb. 6). 
8

$\mathrm{ZB}$

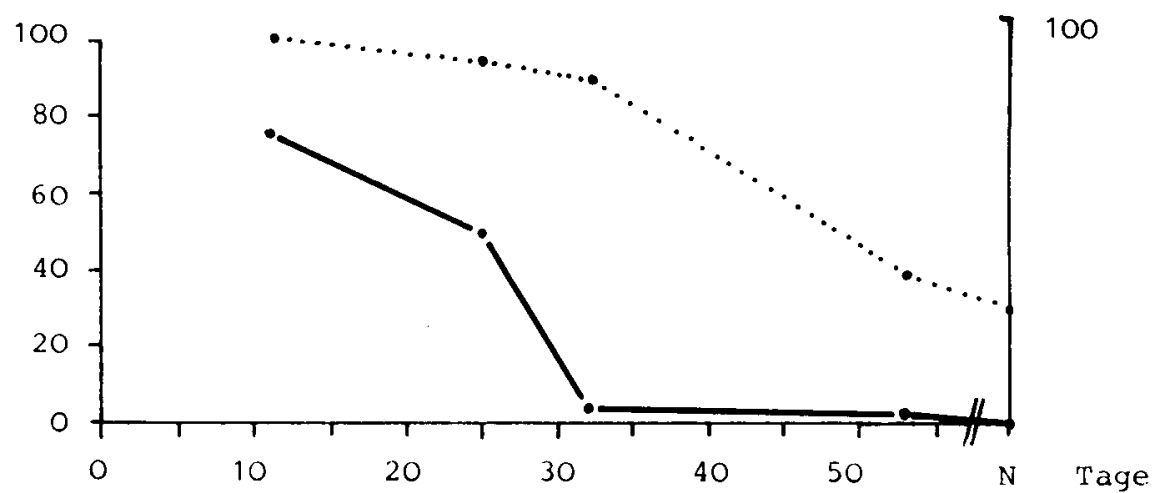

Abb. 6: Merkmal 5 „Implizierte Bewertung“ - relative Häufigkeit von ,negativer Bewertung*

Andererseits nimmt im Zeitverlauf der Anteil der Außerungen zu, in denen die subjektive Bewertung weder der positiven noch der negativen Seite zugerechnet werden kann (s. Abb. 7).

8
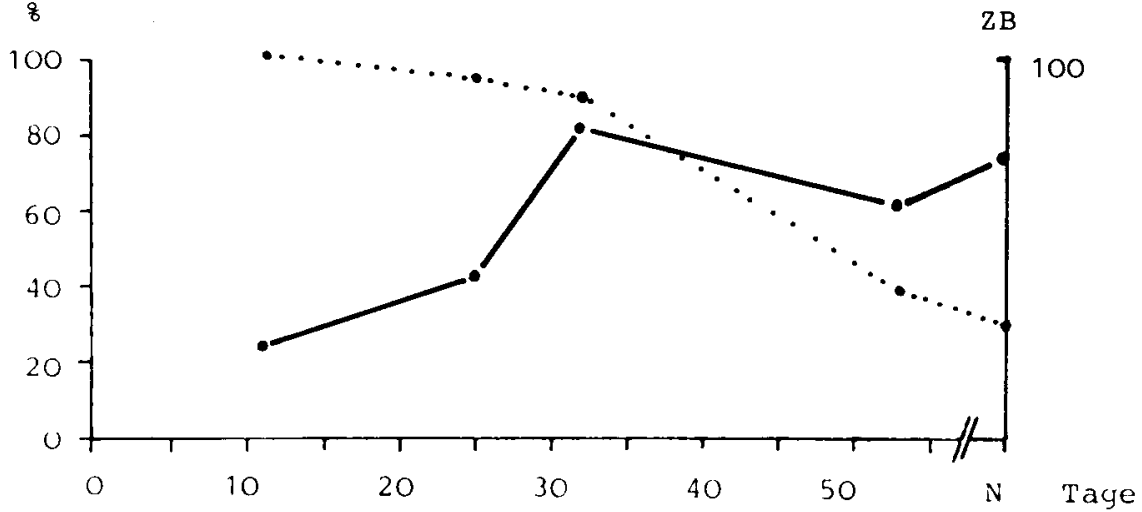

Abb. 7: Merkmal 5 ,Implizierte Bewertung“ - relative Häufigkeit von ,neutraler Bewertung"

Mit zunehmender Besserung des Befindens verändert sich also die subjektive Bewertung des Gesagten durch den Patienten von dem negativen Pol hin zur neutralen Mitte. Unter diesem Aspekt ist auch der Befund zu betrachten, den wir bezüglich der Kategorie „Beruf, Leistung, etc.“ des Merkmals „Thematik " erhielten: Es ergaben sich hier zwar für das erste und dritte Interview etwa die gleichen Prozentanteile, jedoch wurden im ersten Interview alle Äußerungen zu diesem Thema mit einer negativen Bewertung versehen, während derselbe Bereich im dritten Interview neutral gesehen wurde.

„Bezug zur Äußerung des Gesprächspartners":

Mit diesem Merkmal wollten wir vor allem Veränderungen in der Interaktion zwischen den beiden Gesprächspartnern erfassen. 


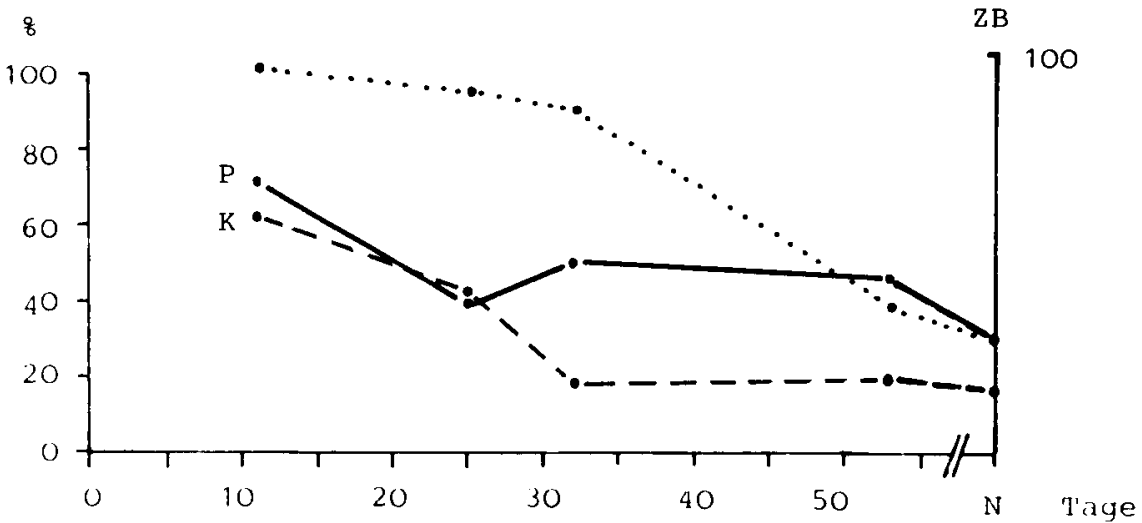

Abb. 8: Merkmal 7 „Bezug zur Äußerung des Gesprächspartners“ - relative Häufigkeit von 7.1. ,deutliche Bezugnahme" für den Kliniker (K) und den Patienten (P)

Die Abbildung 8 zeigt, daß beide Gesprächsteilnehmer darum bemüht sind, in ihren Äußerungen einen Bezug zur vorangegangenen Äußerung des Gesprächspartners herzustellen, jedoch trifft dies für den Patienten in sehr viel stärkerem Maße zu als für den Kliniker. Dagegen zeigt Abb. 9 die im Vergleich zum Patienten stärkeren Tendenzen beim Kliniker, auf die Außerung des Gesprächspartners nicht einzugehen und stattdessen einen neuen Gesichtspunkt einzuführen.

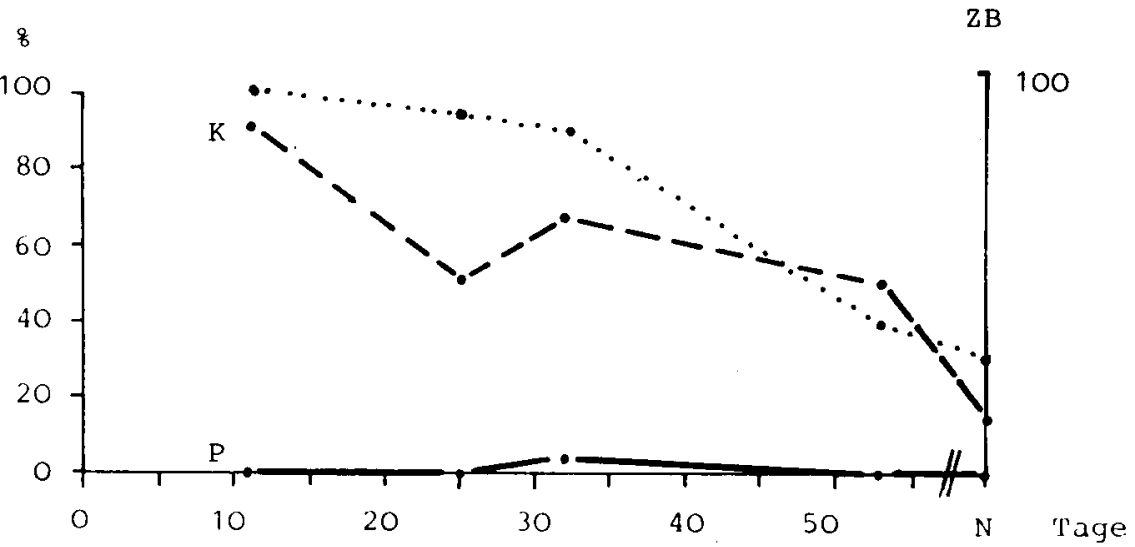

Abb. 9: Merkmal 7 „Bezug zur Äußerung des Gesprächspartners“ - relative Häufigkeit von 7.4. ,keine Bezugnahme, neuer Gesichtspunkt' für K und $\mathrm{P}$

„Äußerungsform“:

Die Abbildung 10 zeigt die stark unterschiedlichen Häufigkeiten von ,,Aussagen, Feststellungen, Behauptungen" für die beiden Teilnehmer. 
客

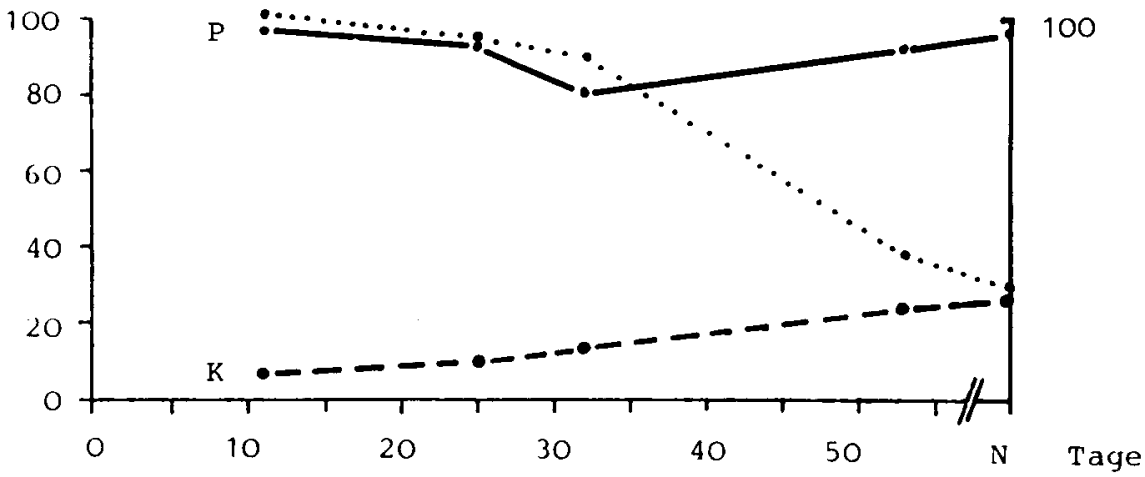

Abb. 10: Merkmal 8 „Äußerungsform“ - relative Häufigkeit von 8.1. ,Aussage etc." für $\mathrm{K}$ und $\mathrm{P}$

Während der Anteil dieser Außerungsform beim Patienten in der Regel um $90 \%$ ausmacht, verwendet der Kliniker diese Form der Äußerung anfänglich kaum, jedoch steigt ihre Häufigkeit mit zunehmender Interviewzahl stetig an (und damit auch im Verlauf der Zustandsbesserung des Patienten).

Dagegen finden wir beim Kliniker einen im Vergleich zum Patienten sehr hohen Anteil an Fragen (Abb. 11).

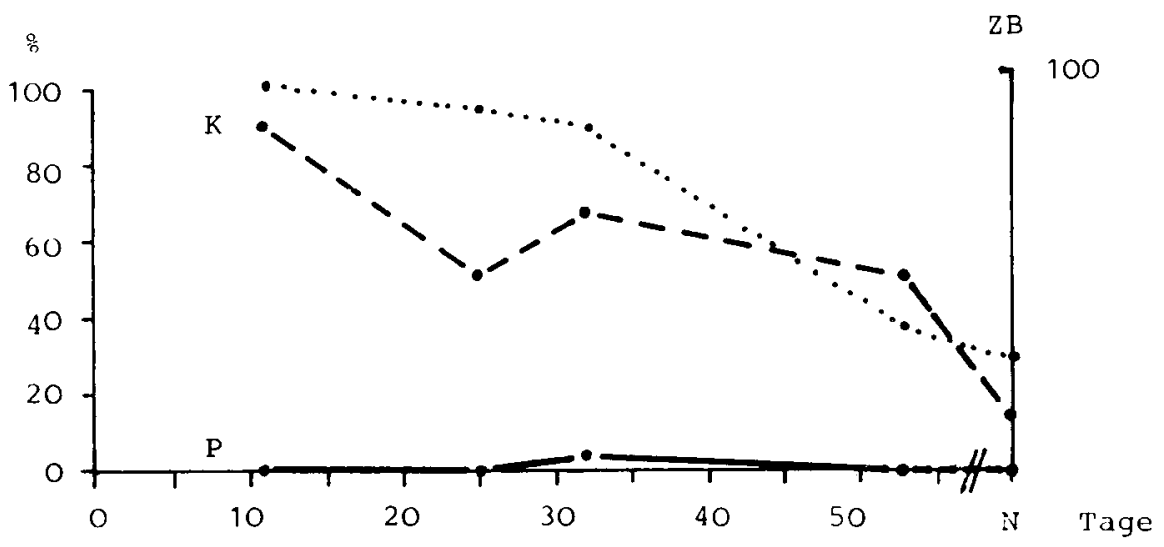

Abb. 11: Merkmal 8 ,Äußerungsform“ - relative Häufigkeit von 8.2. ,Frage“ für K und $\mathrm{P}$

Die Häufigkeit der Fragen geht zwar im Zeitverlauf zurück, um durch Rückmeldungen wie z. B. „hm" oder ,ja" und auch durch Aussagen ersetzt zu werden, auffallend bleibt aber demgegenüber, daß der Patient praktisch keine Fragen stellt. Dieser Befund ist insofern bemerkenswert, als es sich bei den analysierten Gesprächsausschnitten um „freie Gespräche“ handelt, die von dem Standardfragenteil des Interviews abgegrenzt sein sollten. 
Schlußbemerkung:

Nach den bisherigen Resultaten scheint das hier skizzierte Codierungssystem geeignet zu sein, (a) Zustandsveränderungen besonders des Patienten über sein sprachliches Verhalten zu erfassen und (b) die verbale Interaktion zwischen den Gesprächspartnern global zu beschreiben. Es zeigen sich deutliche Veränderungen im Sprachverhalten des Patienten über die Zeit. Weiter scheint sich in der Art ihrer sprachlichen Interaktion eine starke Rollenasymmetrie zwischen Kliniker und Patienten auszudrücken.

Das Verfahren ermöglicht somit diagnostisch eine Verlaufsbeurteilung und bietet möglicherweise auch eine Handhabe, den sprachlichen Ausdruck unterschiedlicher Interview- und Psychotherapieformen quantitativ zu beschreiben.

\section{Literatur}

Beck, A. T., 1967: Depression: Clinical experimental, and theoretical aspects, New York. Brenner, St.-O./Hjelmquist, E., 1978: The production and understanding of utterances in dyadic communication, in: Scandinavian Journal of Psychology 1978, 19, S. $121-$ 131.

Fearing, F., 1967: Toward a Psychological Theory of Human Communication, in: Matson, F. W./Montagu, A., eds., 1967: The Human Dialogue: Perspectives on Communication, New York, S. 179-194.

Gottschalk, L. A./Gleser, G., 1969: The Measurement of Psychological States Through the Content Analysis of Verbal Behavior, Berkeley.

Howard, K. J./Orlinsky, D. E./Hill, J. A., 1969: Content of Dialogue in Psychotherapy, in: Journal of Counseling Psychology 1969, 16, S. 396-404.

Lindenfeld, J., 1972: In search of psychological factors of linguistic variation, in: Semiotica 1972,5 , S. 350-361.

Mahl, G. F., 1956: Disturbances and silences in the patients speech in Psychotherapy, in: Journal of Abnormal Psychology 1956, 53, S. 1-15.

Matarazzo, J. D./Wiens, A.N./Saslow, G. N., 1965: Studies of interview speech behavior, in: Krasner, L./Ullmann, L. P., eds., 1965: Research in Behavior Modification, New York, S. 179-210.

Mintz, J./Luborsky, L., 1970: P-technique factor analyses in psychotherapy: An illustration of a method, in: Psychotherapy, Theory, Research and Practice 1970, 7, S. 13 18.

Weintraub, W./Aronson, H., 1967: The Application of Verbal Behavior Analysis to the Study of Psychological Defense Mechanism. IV. Speech Pattern Associated with Depressive Behavior, in: Journal of Nervous and Mental Disease 1967, 144, S. 22-28.

Weissmann, M. M./Bothwell, S., 1976: Assessment of Social Adjustment by Patient SelfReport, in: Archives of General Psychiatry 1976, 33, S. 1111-1115. 